\title{
Temporal Trends in Prognosis of Patients with Acute Coronary Syndrome Complicated by Ventricular Tachyarrhythmia
}

\author{
Ella Yahud ${ }^{1}$, Avishag Laish Farkash ${ }^{1}$, Nir Shlomo ${ }^{1}$, Noam Fink ${ }^{1}$, Ilan Goldenberg ${ }^{2}$, Eli I. \\ Lev $^{3}$, and Elad Asher ${ }^{4}$ \\ ${ }^{1}$ Affiliation not available \\ ${ }^{2}$ University of Rochester \\ ${ }^{3}$ Assuta Ashdod Hospital \\ ${ }^{4}$ Shaare Zedek Medical Center
}

April 15, 2021

\begin{abstract}
Objectives: To evaluate clinical characteristics and prognosis of patients presented with ventricular tachyarrhythmia (VTA) during the course of acute coronary syndrome (ACS) and to analyze it according to period of presentation. Background: VTA is an infrequent yet serious complication of ACS. There is limited data regarding the incidence and prognostic implications of VTA in the last decade as compared with the previous decade. Methods: We evaluated clinical characteristics, major adverse cardiovascular events, short and long- term mortality of patients hospitalized with ACS who were enrolled in the Acute Coronary Syndrome Israeli Survey (ACSIS) during the years 2000-2016. Patients were classified into three groups: no VTA, early VTA ([?]48h of onset) and late VTA ( $>48 \mathrm{~h}$ of onset). Data were analyzed according to decades of presentation (current decade vs. previous decade). Results: The study population comprised 15,200 patients. VTA occurred in 487 (3.2\%) of patients. Early VTA presented in $373 / 487(77 \%)$ patients and late VTA in 114/487 (23\%) patients. VTA's, occurring in ACS patients were associated with increased risk of in-hospital, 30-days, 1-year and 5-year mortality rates during both early and late periods in compared with no VTA. Moreover, late VTA was associated with the highest mortality rate with up to $65 \%$ in 5 -year follow up $(\mathrm{p}<0.001)$. Nevertheless, late VTA was associated with lower mortality rate in the current decade (2008-2016) compared with last decade (2000-2006). Conclusions: Any VTA following ACS was associated with high short and long-term mortality rate. However, over the past decade there has been a significant improvement in survival rates, especially in patients with late VTA. This may be attributed to early and invasive reperfusion therapy, implantable cardioverter defibrillator implantation and better medical treatment.
\end{abstract}

\section{Temporal Trends in Prognosis of Patients with Acute Coronary Syndrome Complicated by Ventricular Tachyarrhythmia}

Ella Yahud $\mathrm{MD}^{1}$, Avishag Laish-Farkash MD, $\mathrm{PhD}^{1}$, Nir Shlomo $\mathrm{MSc}^{2}$, Noam Fink $\mathrm{MD}^{2}$, Ilan Goldenberg $\mathrm{MD}^{3}$, Eli Lev MD ${ }^{1}$, Elad Asher MD, MHA ${ }^{4}$

${ }^{1}$ Cardiology Division, Assuta Ashdod University Hospital, Israel

${ }^{2}$ Leviev Heart Center, Sheba Medical Center, Tel Hashomer, Israel

${ }^{3}$ University of Rochester Medical Center, Rochester, NY, USA

4 Heart Institute, Shaare Zedek Medical Center, Hebrew University, Jerusalem, Israel

\section{Corresponding Author:}

Ella Yahud, MD 
Assuta Ashdod University Hospital

7747629 Ha-Refua St 7, Ashdod, Israel

Tel: +972523935075

Fax: +972737436226

Email: ellaya@assuta.co.il

No conflict of interest and no relationship with industry exists

Abstract:

Objectives: To evaluate clinical characteristics and prognosis of patients presented with ventricular tachyarrhythmia (VTA) during the course of acute coronary syndrome (ACS) and to analyze it according to period of presentation.

Background: VTA is an infrequent yet serious complication of ACS. There is limited data regarding the incidence and prognostic implications of VTA in the last decade as compared with the previous decade.

Methods: We evaluated clinical characteristics, major adverse cardiovascular events, short and long- term mortality of patients hospitalized with ACS who were enrolled in the Acute Coronary Syndrome Israeli Survey (ACSIS) during the years 2000-2016. Patients were classified into three groups: no VTA, early VTA ([?]48h of onset) and late VTA ( $>48 \mathrm{~h}$ of onset). Data were analyzed according to decades of presentation (current decade vs. previous decade).

Results: The study population comprised 15,200 patients. VTA occurred in $487(3.2 \%)$ of patients. Early VTA presented in 373/487 (77\%) patients and late VTA in 114/487 (23\%) patients. VTA's, occurring in ACS patients were associated with increased risk of in-hospital, 30-days, 1-year and 5-year mortality rates during both early and late periods in compared with no VTA. Moreover, late VTA was associated with the highest mortality rate with up to $65 \%$ in 5 -year follow up $(\mathrm{p}<0.001)$. Nevertheless, late VTA was associated with lower mortality rate in the current decade (2008-2016) compared with last decade (2000-2006).

Conclusions: Any VTA following ACS was associated with high short and long-term mortality rate. However, over the past decade there has been a significant improvement in survival rates, especially in patients with late VTA. This may be attributed to early and invasive reperfusion therapy, implantable cardioverter defibrillator implantation and better medical treatment.

Key words: Ventricular tachyarrhythmia, all-cause mortality, acute coronary syndrome, NSTEMI, STEMI, risk-factors.

What is already known about this tt does this article add?

What is known about this topic?

VTA is rare yet serious complication of acute coronary syndrome (ACS) and has adverse implications on in-hospital and long-term mortality based on older trials. VTA associated with acute myocardial infarction (AMI) are classified as early (within the first 48 hours) which are the most frequent cause of AMI-related death and late (after 48 hours), usually associated with ventricular dysfunction. Limited data exists regarding the association between VTA observed during AMI hospitalization and short and long-term mortality.

What does this study contribute to the literature?

In the current study, majority of VTA occurred during the first 48 hours of ACS. The incidence of both early and late VTA did not change significantly during the years 2000-2016, suggesting it was not related to medical treatment. The overall prognosis of ACS patients improved in the last decade and the most prominent improvement was seen in those who had late VTA.

\section{Introduction}


Ventricular tachyarrhythmia (VTA) especially sustained ventricular tachycardia and ventricular fibrillation, is an infrequent yet serious complication of acute coronary syndrome (ACS). Prior studies reported that VTA complicates $2-8 \%$ of ACS cases and has adverse implications on in-hospital and long-term mortality $(1,2)$. Early VTAs occur in the first 48 hours of acute myocardial infarction (AMI) and are the most frequent cause of AMI-related death (3). Late VTAs i.e. > 48 hours of AMI onset are usually associated with ventricular dysfunction (4). Over the past decade, major advances in the treatment of patients with AMI have occurred and led to improved survival (5). Currently, however, there is limited data regarding the incidence and temporal trends over recent years in the outcomes of patients presenting with VTA complicating ACS. Moreover, limited information exists regarding the association between VTA observed during ACS hospitalization to short and long-term mortality. This is despite overall enhanced therapy and improved survival among ACS patients. Therefore, the purpose of the current study was to evaluated temporal trends of clinical characteristics and outcomes over the last two decades (2000-2016) among ACS patients presenting with and without VTA who were enrolled in the Acute Coronary Syndrome Israeli Survey (ACSIS) survey.

\section{Methods}

\section{Study population}

The present study is a cohort data analysis of patients who were enrolled prospectively in the ACSIS from 2000 to 2016. Details of ACSIS have been previously reported (6). In brief, this survey is conducted biennially over a 2-month period among ACS patients admitted to coronary care units and cardiology wards in all 27 public hospitals in Israel.

Patient management was at the discretion of the attending physicians. The diagnosis of AMI and STsegment elevation MI (STEMI) or non- ST-segment elevation MI (NSTEMI), was made by the attending cardiologist according to pre-specified survey criteria based on accepted guidelines. In total 15,200 patients were enrolled in our study. The patients were grouped according to the occurrence of ventricular arrhythmias during the course of index AMI. Data included baseline demographics, medical history, hospital course, procedures, complications, and outcome based on hospital's charts. Additional data regarding health status, adverse events and medication adherence were collected from the patients via follow-up calls and from outpatients' medical records one month after hospital discharge. Mortality rates were determined for all patients from hospital and out-patient charts and by matching their identification numbers with the Israeli National Population Register. In- hospital, one year, and five-year all-cause mortality was assessed for the absence of VTA, early VTA and late VTA according to the periods of presentation.

\section{Definitions and endpoints}

The diagnosis of VTA, sustained ventricular tachycardia or ventricular fibrillation was based on electrocardiogram (ECG) or continuous monitor strips. Sustained ventricular tachycardia was defined as a series of abnormally shaped QRS complexes longer than 120 milliseconds, with a rate 100-250 beats/minute, lasting longer than 30 seconds or requiring electrical cardioversion due to hemodynamic collapse. Ventricular fibrillation was defined as rapid, very irregular rhythm with indiscernible P waves or QRS. Patients were classified into three groups: no VTA; early VTA ([?]48h of onset) and late VTA ( $>48 \mathrm{~h}$ of onset). Survey periods were divided to early decade (2000-2006) vs. late decade (2008-2016). Primary endpoints were 30-day, 1-year and 5-year mortality rate. Secondary endpoints included 30-day major adverse cardiac events (MACE) (all-

cause mortality rate, re-infarction, stent thrombosis and urgent revascularization). Left ventricular ejection fraction (LVEF) was graded according to echocardiography performed at admission in each center.

\section{Statistical methods}

Demographic, clinical features and the use of in-hospital therapies were reported for all study participants. Multiple logistic regression adjustments for age, gender, diabetes mellitus, chronic renal failure and heart 
failure and for survey periods was performed to assess the adjusted odds ratio (OR) and $95 \%$ confidence interval (CI) of in and out of hospital complications and 30-day, 1 and 5-year mortality rate associated with VTA at any time during and after the admission for ACS. The cumulative probability of all-cause mortality rate was graphically displayed using the Kaplan-Meier method and compared using the log-rank test. In order to evaluate the independent association of VTA with the 5-year all-cause mortality rate outcome, a Cox proportional hazards model was constructed adjusting for survey period. We employed an interactionterm analysis to examine whether the risk of 5-year mortality rate associated with VTA was different in the early period as compared with the late period. Statistical significance was accepted for a 2 -sided $\mathrm{p}<0.05$. Statistical analysis was performed using the R Statistical Package (R Foundation for Statistical Computing, Vienna, Austria).

\section{Results}

Baseline characteristics

The study population comprised of 15,200 patients with ACS. Of them 11682 (77\%) were males, 5383 (35\%) suffered from diabetes mellitus 8894 (59\%) had a history of arterial hypertension, $9757(64 \%)$ had dyslipidemia and 6931 (46\%) presented with STEMI. Patient's baseline characteristics are presented in Table 1 .

Ventricular tachyarrhythmia characteristics

Four hundred eighty-seven patients $(3.2 \%)$ had VTA during their hospitalization. Among those patients, $373 / 487$ (77\%) had early VTA and 114/487 (23\%) had late VTA. The incidence of both types of VTA's remained the same in the past 15 years as shown in Figure 1. Patients with late VTA were older (65+-14 vs. $64+-13, \mathrm{p}<0.001)$ and had significantly higher incidence of co-morbidities and coronary risk factors such as diabetes mellitus, arterial hypertension, as well as known coronary artery disease, and chronic renal failure as shown in Table 1.

\section{Outcome}

Rate of MACE was higher in late VTA then in early and non-VTA (49\% vs. $23 \%$ vs. $8 \%$, respectively, $\mathrm{p}<0.001)$. As shown with logistic regression model the presence of late VTA was associated with three folds' higher ratios for 30 days MACE $(\mathrm{p}<0.001)$, Figure 2. In- hospital and 1-year mortality rate were also higher in the presence of late VTA as compared with no VTA [HR 27.44 (95\% CI 17.56- 42.49) and 9.78 (95\% CI 6.57-11.18), respectively, $\mathrm{p}<0.001]$. Even after multivariate logistic regression for confounders the presence of early VTA and late VTA, were independently associated with increased risk of in-hospital mortality rate [OR 9.65 (95\% CI 7.0-13.13) and 27.64 (95\% CI 17.56-42.49), respectively, $\mathrm{p}<0.001$ ], and early postdischarge mortality rate [OR 8.75 (95\% CI 5.98-12.24) and 22.6 (95\% CI 14.5-35.62), respectively, p<0.001]. Moreover, multivariable Cox proportional hazard analysis including baseline potential confounders confirmed that 5-year mortality rate was also higher in late VTA then in early and non-VTA (HR 8.27 vs. 2.65, for late and early VTA, respectively $95 \%$ CI 2.05-3.42).

Late vs. Early period

The 1 and 5-year all-cause mortality rate was lower during the late period as compared with the early period for patients with no VTA, early VTA and late VTA ( $8 \%$ vs $10 \%, 20 \%$ vs $25 \%$ and $37 \%$ vs $65 \%$, all log-rank p $<0.001$, respectively for 1 year) and (20\% vs $23 \%, 26 \%$ vs $29 \%$ and $45 \%$ vs $75 \%$, all log-rank p <0.001, respectively for 5 years) as shown in Figure 3 and 4 . Moreover, the late period was associated with lower all-cause mortality rates for in-hospital, 1 year and 5 years mortality rate (OR 0.56, 0.66 and $0.82, \mathrm{p}<0.001$, respectively).

In the early period ICD was implanted during hospitalization in $15(0.2 \%)$ patients with no VTA, $3(1.4 \%)$ patients with early VTA and $5(8 \%)$ with late VTA $(\mathrm{p}<0.001)$. In the late period in hospital implantation of ICD was performed in $25(0.4 \%), 8(5 \%)$ and 0 patients, respectively $(\mathrm{p}<0.001)$. 
In our study during early period chronic treatment with beta blockers was administered to $34 \%, 24 \%$ and $42.5 \%$ of patients with no VTA, early VTA and late VTA respectively, $\mathrm{p}=0.018$; beta blockers at discharge were prescribed to $78 \%, 72 \%$ and $51 \%$ of patients respectively, $\mathrm{p}<0.001$

During the late period $38.5 \%, 35 \%$ and $42 \%$ of patients with no VTA, early VTA and late VTA, respectively, used beta blockers chronically, $\mathrm{p}=0.6$ and $80.5 \%, 81.5 \%, 73 \%$ of patients, respectively, were discharged with beta blockers, $\mathrm{p}=0.4$ Chronic angiotensin-converting-enzyme inhibitor (ACEI) treatment in early period was used in $26 \%, 31 \%$ and $22 \%$ of patients with no VTA, early VTA and late VTA $\mathrm{p}=0.8$; ACEI after discharge were prescribed to $61 \%, 61.5 \%$ and $57 \%$, respectively, $\mathrm{p}=0.9$

During the late period chronic ACEI treatment was administered to $32 \%, 32 \%, 24 \%$ of patients with no VTA, early VTA and late VTA respectively, $\mathrm{p}=0.08$; ACEI in discharge were prescribed to $67 \%, 66 \%, 47 \%$ of patients respectively, $\mathrm{p}=0.018$

\section{Discussion}

In the current study, VTA occurred in roughly $3 \%$ of ACS patients admitted to the hospital with the majority (77\%) of those arrhythmias occurred during the first 48 hours of ACS. Moreover, the incidence of both early and late VTA did not change significantly during the years 2000-2016, suggesting it was not related to medical treatment.

Previous data on prognostic significance of VTA is controversial. In the HORIZONS-AMI Trial (7) there was no significant difference in MACE and 3-year mortality rate in patients with or without VTA that occurred after primary percutaneous coronary intervention (PCI). Another recent study, found that in patients with STEMI that survived out of hospital cardiac arrest due to VTA and underwent primary PCI, the 1-year mortality was equal to those without out of hospital cardiac arrest (8). On the other hand, in the MILIS study (9) the in hospital mortality rate was significantly increased by VTA, especially late VTA, causing cardiac arrest during ACS hospitalization, although there was no difference in mortality rate in the 32-month follow up as compared with patients with no VTA. In APEX AMI trial (10), pre and post primary PCI VTA were also shown to be associated with significantly increased 90 days mortality. Piccini et al. (11) studied a large population of 9000 patients with STEMI and NSTEMI undergoing PCI. In their study, the rate of early VTA was $5.2 \%$ and it was also related to increase mortality risk by 4.4 folds as compared with no VTA. Our data is in accord to the aforementioned trials suggesting that VTA occurrence during the course of ACS is not benign. Short and long-term mortality rate was increased dramatically by the presence of early and especially late VTA as compared with no VTA. This was consistent even after adjustment for multiple confounders. In the logistic regression analysis model the presence of late VTA was associated with 27 folds' increase of in hospital mortality, 10 folds increased one-year mortality and 8 folds increased 5 years mortality rate. The proposed reason why late VTA carries an increased risk of death might be its association with pump failure and hemodynamic deterioration rather than arrhythmic death $(12,13)$.

Interestingly, although the overall prognosis of ACS patients improved in the last decade (14) as well as in our study, the most prominent improvement in our study was seen in those who had late VTA, as shown in Figures 3 and 4. Wider use of ACE inhibitors and beta blockers at discharge in the late VTA group, early reperfusion strategy, $(15,16)$, as well as wider use of implantable and wearable cardioverter defibrillators could explain our findings.

\section{Limitations}

Due to its observational non-randomized design, the current study is subject to limitations as described in detail previously (17). Thus, despite efforts to control for confounding factors by applying multivariate analysis, we cannot exclude unmeasured factors, which could have biased the results. Moreover, data regarding 5 years survival rate is limited in the late period with no long term data since the year 2016. Nevertheless, in spite of these limitations, the study in a multicenter nationwide survey with large study population and long term outcomes.

\section{Conclusions}


In summary, in this national, multicenter, contemporary "real life" setting, we did not observe any change in the rate of VTA. Nevertheless, any VTA following AMI was associated with high short and long-term mortality rate. However, over the past decade there has been a significant improvement in survival rates, especially in patients with late VTA that may be attributed to early and invasive reperfusion therapy, implantable cardioverter defibrillator implantation and better medical treatment.

This statement is to certify that all authors have seen and approved the manuscript

being submitted, have contributed significantly to the work, attest to the validity and legitimacy of the data and its interpretation, and agree to its submission to the International Journal of Clinical Practice.

The article is the Authors' original work, has not received prior publication and is not under consideration for publication elsewhere.

All the authors report no relationships that could be construed as a conflict of interest.

References:

1. Metha RH, Starr AZ, Lopes RD, et al. Incidence of and outcomes associated with ventricular tachycardia or fibrillation in patients undergoing primary percutaneous coronary intervention, JAMA 2009, vol.301 (pg.1779-1789).

2. Al-Khatib SM, Granger CB, Huang Y, et al. Sustained ventricular arrhythmias among patients with acute coronary syndromes with no ST-segment elevation: incidence, predictors, and outcomes, Circulation 2002, vol. 106 (pg.309-312)

3. Liang JJ, Hodge DO, Metha RA, Russo AM, Prasad A, Cha YM. Outcomes in patients with sustained ventricular tachyarrhythmias occurring within $48 \mathrm{~h}$ of acute myocardial infarction: when is ICD appropriate? Europace 2014; 16:1759-66

4. Volpi A, Cavalli A, Santoro E, Tognoni G. Incidence and prognosis of secondary ventricular fibrillation in acute myocardial infarction. Evidence for a protective effect of thrombolytic therapy. GISSI Investigators. Circulation 1990; 82:1279-88

5. Yeh RW, Sidney S, Chandra M, Sorel M, Selby JV, Go AS. Population trends in the incidence and outcomes of acute myocardial infarction. N Engl J Med 2010; 362: 2155-2165.

6. Asher E, Abu-Much A, Goldenberg I, et al. Platelets and Thrombosis in Sheba-PLATIS Study Group. Incidence and Clinical Features of Early Stent Thrombosis in the Era of New P2y12 Inhibitors (PLATIS-2). PLoS One 2016;11(6):e0157437

7. Metha RH, Yu J, Piccini JP, et al. Prognostic significance of postprocedual sustained ventricular tachycardia or fibrillation in patients undergoing primary percutaneous coronary intervention (from the HORIZONSAMI Trial). Am J Cardiol 2012;109(6):805-12

8. Demirel F, Rasoulm S, Elvan A, et al. Impact of out-of-hospital cardiac arrest due to ventricular fibrillation in patients with ST- elevation myocardial infarction admitted for primary percutaneous coronary intervention: Impact of ventricular fibrillation in STEMI patients. Eur Heart J Acute Cardiovasc Care 2015;4(1):16-23.

9. Tofler GH, Stone PH, Muller JE, et al. Prognosis after cardiac arrest due to ventricular tachycardia or ventricular fibrillation associated with acute myocardial infarction (MILIS Study). Multicenter investigation of the limitation of infarct size. Am J Cardiol 1987; 60(10):755-61

10. Metha RH, Starr AZ, Lopes RD, et al. Incidence of and outcomes associated with ventricular tachycardia or fibrillation in patients undergoing primary percutaneous coronary intervention. JAMA 2009; 301(17):177989. 
11. Piccini JP, Berger JS, Brown DL. Early sustained ventricular arrhythmias complicating acute myocardial infarction. Am J Med 2008; 121(9):797-804.

12. Newby KH, Thompson T, Stebbins A, Topol EJ, Califf RM, Natale A. Sustained ventricular

arrhythmias in patients receiving thrombolytic therapy: incidence and outcome. The GUSTO investigators. Circulation 1998; 98(23):2567-73.

13. Bulent Gorenek, Carina Blomstrom Lundqvist, Josep Brugada Terradellas, et al. ESC Scientific Document Group. Cardiac arrhythmias in acute coronary syndromes: position paper from the joint EHRA, ACCA, and EAPCI task force.

EP Europace 2014; 16(11):1655-1673.

14. Moran AE, Forouzanfar MH, Roth GA, et al. Temporal trends in ischemic heart disease mortality in 21 world regions, 1980 to 2010: the Global Burden of Disease 2010 study. Circulation 2014;129(14):1483-92.

15. Asher E, Tal S, Mazin I, et al. Effect of Chewing vs Swallowing Ticagrelor on Platelet Inhibition in Patients With ST-Segment Elevation Myocardial Infarction: A Randomized Clinical Trial. JAMA Cardiol 2017;2(12):1380-1384.

16. Asher E, Frydman S, Katz M, et al. Chewing versus Swallowing Ticagrelor to Accelerate Platelet Inhibition in Acute Coronary Syndrome - the CHEERS study. For The PLATIS (Platelets and Thrombosis in Sheba) Study Group. Thromb Haemost 2017;117(4):727-733

17. Asher E, Fefer P, Sabbag A, et al. Prior chronic clopidogrel therapy is associated with increased adverse events and early stent thrombosis. Thromb Haemost 2016; 115 (2):433-8.

Table 1: Baseline characteristics by VTA presence

\begin{tabular}{|c|c|c|c|c|}
\hline & $\begin{array}{l}\text { No VTA } \\
\mathrm{N}=14713\end{array}$ & $\begin{array}{l}\text { Early VTA } \\
\mathrm{N}=373\end{array}$ & Late VTA $N=114$ & p-value \\
\hline Age $( \pm S D)$, years & $64 \pm 13$ & $60 \pm 12$ & $65 \pm 14$ & $<0.001$ \\
\hline Male gender, \% & $11287(77)$ & $308(83)$ & $87(76)$ & 0.030 \\
\hline $\begin{array}{l}\text { Weight }(\mathrm{kg}) \\
( \pm \mathrm{SD})\end{array}$ & $79 \pm 15$ & $80 \pm 17$ & $83 \pm 17$ & 0.162 \\
\hline $\begin{array}{l}\text { Diabetes Mellitus, } \\
\%\end{array}$ & $5256(36)$ & $89(24)$ & $38(33)$ & $<0.001$ \\
\hline Hypertension, \% & $8652(59)$ & 171(46) & $71(62)$ & $<0.001$ \\
\hline Dyslipidemia, \% & $9505(65)$ & $195(53)$ & $57(51)$ & $<0.001$ \\
\hline $\begin{array}{l}\text { Past stroke/TIA, } \\
\%\end{array}$ & $1186(8)$ & $31(8)$ & $5(4)$ & 0.355 \\
\hline $\begin{array}{l}\text { Prior Chronic } \\
\text { Renal Failure, \% }\end{array}$ & $11612(11)$ & $20(5)$ & $120(17)$ & $<0.001$ \\
\hline Prior COPD, \% & $697(6)$ & $23(7.9)$ & $3(4)$ & 0.455 \\
\hline Prior MI, \% & $4481(31)$ & $102(28)$ & $38(33)$ & 0.360 \\
\hline $\begin{array}{l}\text { Prior AP in } 24 \\
\text { hours, } \%\end{array}$ & $5168(35)$ & $77(21)$ & $41(36)$ & $<0.001$ \\
\hline Prior CABG, \% & 1481(10) & $15(4)$ & $8(7.1)$ & $<0.001$ \\
\hline History of PCI, \% & $4058(28)$ & $77(21)$ & $28(25)$ & 0.009 \\
\hline Heart failure, $\%$ & $1144(8)$ & $29(8)$ & $15(13)$ & 0.106 \\
\hline $\begin{array}{l}\text { Family history of } \\
\text { CAD, } \% \\
\text { Clinical } \\
\text { Presentation }\end{array}$ & $3466(25)$ & $74(21)$ & $31(29)$ & 0.214 \\
\hline
\end{tabular}




\begin{tabular}{|c|c|c|c|c|}
\hline & $\begin{array}{l}\text { No VTA } \\
\mathrm{N}=14713\end{array}$ & $\begin{array}{l}\text { Early VTA } \\
\mathrm{N}=373\end{array}$ & Late VTA $N=114$ & p-value \\
\hline STEMI, \% & $6564(45)$ & $289(78)$ & $78(68)$ & $<0.001$ \\
\hline $\begin{array}{l}\text { Heart Rate } \\
\text { (mean } \pm \mathrm{SD}), \mathrm{bpm} \\
\text { Treatments }\end{array}$ & $80 \pm 20$ & $87 \pm 29$ & $89 \pm 24$ & $<0.001$ \\
\hline $\begin{array}{l}\text { Primary } \\
\text { Reperfusion, \% }\end{array}$ & $4458(30)$ & $249(67)$ & $53(47)$ & $<0.001$ \\
\hline $\begin{array}{l}\text { Coronary } \\
\text { angiography (till } \\
\text { hospital } \\
\text { discharge),\% }\end{array}$ & $9513(86)$ & $224(89)$ & $69(81)$ & 0.215 \\
\hline PCI, $\%$ & $5862(40)$ & $89(24)$ & $40(35)$ & $<0.001$ \\
\hline $\begin{array}{l}\text { Emergency } \\
\text { CABG, } \%\end{array}$ & $700(5)$ & $11(3)$ & $6(5)$ & 0.250 \\
\hline $\begin{array}{l}\text { Beta Blockers } \\
\text { chronic, } \%\end{array}$ & $4664(36)$ & $90(30)$ & $38(42)$ & 0.029 \\
\hline $\begin{array}{l}\text { Beta Blockers at } \\
\text { discharge, } \% \\
\text { Outcome }\end{array}$ & $11347(79)$ & $258(76)$ & $55(62)$ & $<0.001$ \\
\hline $\begin{array}{l}\text { MACE in } 30 \\
\text { days, } \%\end{array}$ & $1173(8)$ & $84(23)$ & $56(49)$ & $<0.001$ \\
\hline $\begin{array}{l}\text { All-cause } \\
\text { mortality } 30 \text { days, } \\
\%\end{array}$ & $625(4)$ & $69(19)$ & $49(43)$ & $<0.001$ \\
\hline $\begin{array}{l}\text { All-cause } \\
\text { mortality } 1 \text { year, } \\
\%\end{array}$ & $1208(9)$ & $76(23)$ & $55(53)$ & $<0.001$ \\
\hline $\begin{array}{l}\text { All-cause } \\
\text { mortality } 2 \text { years, } \\
\%\end{array}$ & 1596(13) & $83(26)$ & $60(59)$ & $<0.001$ \\
\hline
\end{tabular}

VTA- Ventricular tachyarrhythmia, SD- standard deviation, PCI - percutaneous coronary intervention, CABG - coronary artery bypass grafting, STEMI -ST elevation myocardial infarction, CAD- coronary artery disease, MACE- major adverse cardiac events, TIA - transient ischemic attack, PAD- peripheral artery disease, COPD- chronic obstructive pulmonary artery

disease.

Figure 1. Trends in VTA during the years 2000-2016 


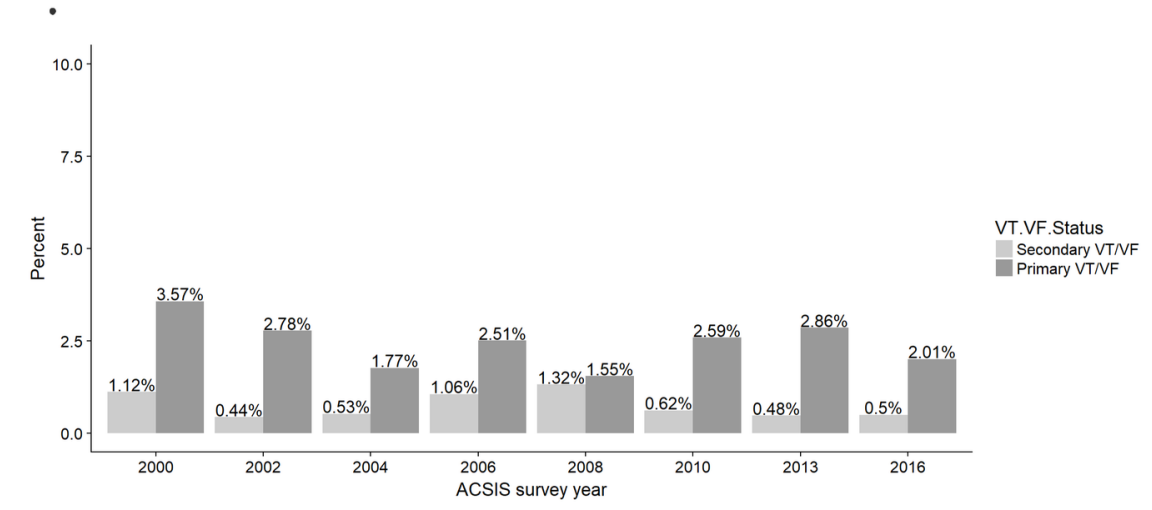

\section{Early VTA}

Late VTA

Figure 2. Major adverse cardiac events (MACE) at 30 days for early and late VTA

OR for 30 day MACE with $95 \% \mathrm{Cl}$

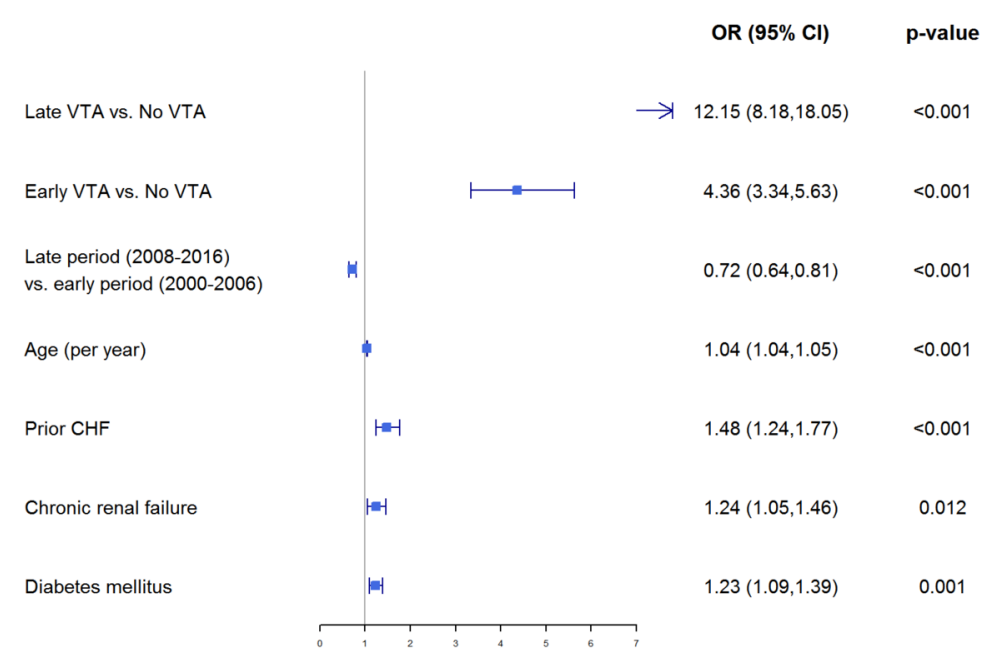

*MACE- all-cause mortality rate, re-infarction, stent thrombosis and urgent revascularization $\mathrm{CI}=$ confidence interval, $\mathrm{OR}=$ odds ratio

Figure 3. One-year survival rate according to period of presentation 


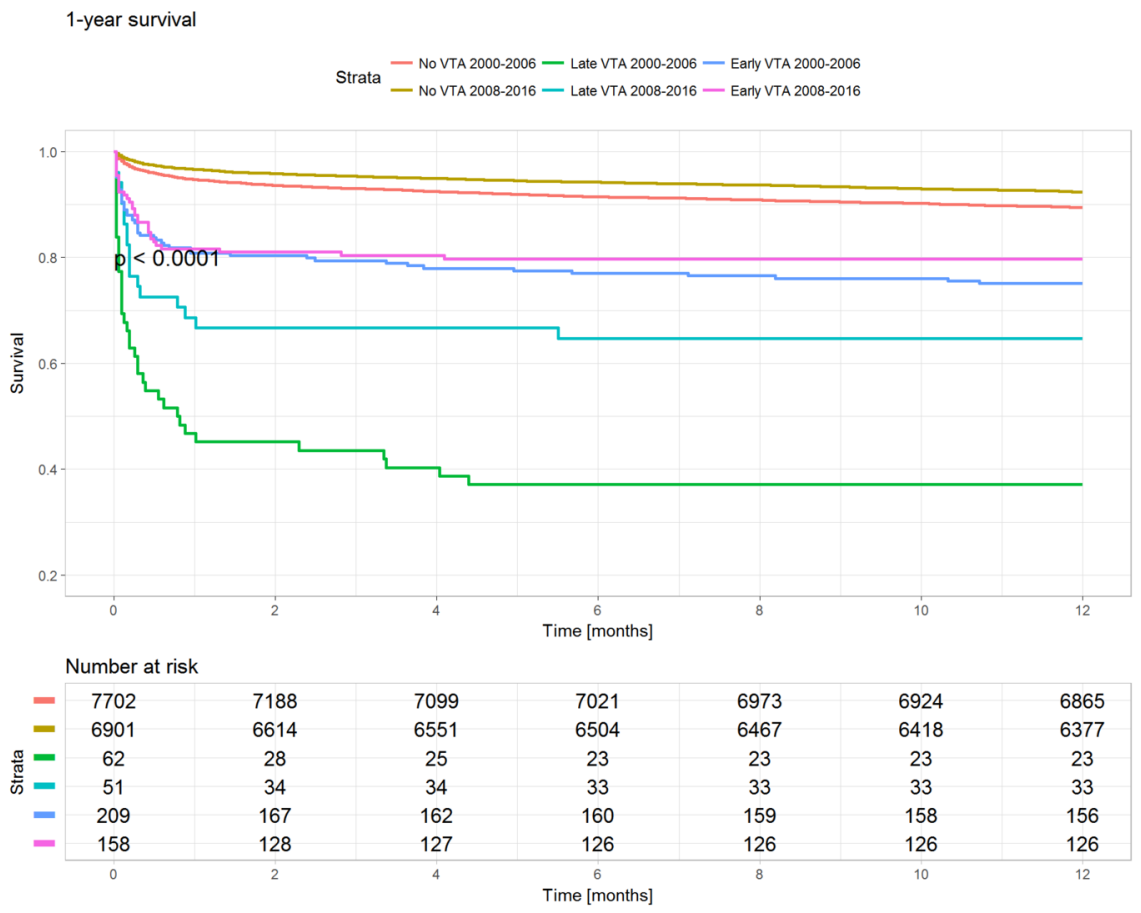

Figure 4. Five-year survival according to period of presentation

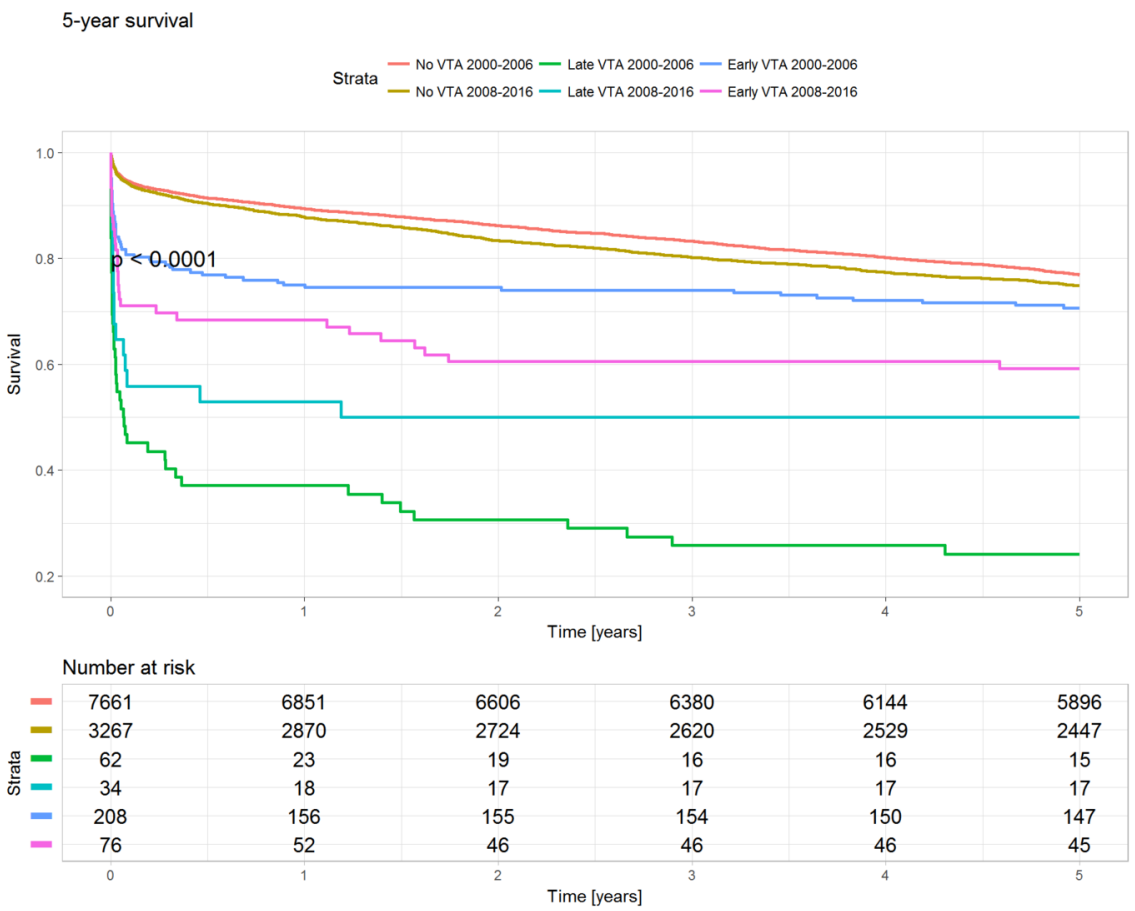

